\title{
An Exploration of the Enablers and Barriers to the Implementation of the Scottish Stroke Care Bundle.
}

\author{
Iona Lancaster ${ }^{1}$ and Linda Martindale ${ }^{2}$ \\ ${ }^{1}$ NHS Tayside \\ ${ }^{2}$ University of Dundee
}

May 18, 2020

\begin{abstract}
Rationale, aims and objectives The Scottish Stroke Care Bundle (SSCB) aims to significantly improve patient outcomes and the consistency with which stroke patients are managed. The identification of key enablers and barriers to implementation will allow a future focus on the development of appropriate interventions to promote reliability of implementation. The aim of this study was to explore and describe the reasons why doctors and nurses in one Health Board area in Scotland feel that the SSCB is not being applied in a consistent and reliable way. The research objective was 'to explore and describe the factors associated with compliance and non-compliance with the Scottish stroke care bundle'. Method A qualitative approach was used to understand the perspectives of medical and nursing staff involved in implementing the SSCB. Eight semi-structured interviews were undertaken across two sites. Cane et al's Theoretical Domains Framework (TDF) guided data collection and analysis providing context-based explanations as to why reliable implementation of the SSCB is not being achieved.1 Results Three significant factors were identified that significantly influence the likelihood of patients receiving the SSCB: knowledge; resources; and organisational culture. The interrelationships between these factors revealed key enablers and barriers to the consistent application of the SSCB in this Health Board area. Conclusions Many of the findings are commonly identified as being crucial to the implementation of care bundles and potentially have national implications for SSCB implementation. Multifaceted quality improvement interventions, based on these findings, would improve the consistency of SSCB implementation in this Health Board area.
\end{abstract}

\section{Introduction}

Stroke is the third commonest cause of death in Scotland; It is a leading cause of disability and causes a greater range of disabilities than any other. ${ }^{2}$ In Scotland stroke is a national clinical priority and is supported by a suite of evidence based clinical standards aimed at promoting continual improvements to care. Performance is monitored both locally and nationally by the Scottish Stroke Care Audit (SSCA).

Based on emerging evidence of the most important drivers for improving stroke outcomes in 2014 the Scottish Government introduced a national stroke 'bundle of care' ${ }^{3}$ In 2016 the SSCB standard was introduced ${ }^{4}$ with the aim that " $80 \%$ of all patients admitted to hospital with a diagnosis of stroke should receive the appropriate elements of the stroke care bundle." To date no Health Board has managed to consistently achieve this target with the latest published information showing a national average of $68 \%$ attainment. ${ }^{5}$

A literature review was undertaken to underpin the study, but no evidence was found which related to the barriers or enablers to the implementation of the SSCB. Identification of local enablers and barriers was identified as potentially valuable for informing improvement activities relating to compliance with the SSCB, so the aim of the study was to explore and describe the reasons why medical and nursing staff in one Scottish Health Board area felt that the SSCB was not being applied in a consistent and reliable way

Method 
As the study was exploratory, descriptive and contextual in nature a qualitative study was undertaken, using in-depth, semi-structured interviews. Responsibility for implementation of the SSCB lies with nursing and medical staff, so the convenience sample included both groups. The setting for the study was two hospitals in one Health Board, where stroke patients are admitted via acute medical units (AMU) to their respective stroke units. In both hospitals' implementation of the SSCB is usually coordinated by AMU or Stroke Unit staff. Therefore participants had to have a minimum of six months' experience of working in either area to ensure they could respond with detailed information about their experience of SSCB implementation. Posters, flyers and ward meetings were used to recruit eight participants; two medical and two nursing staff from each hospital. Data was collected from participants using one-to-one interviews over a period of 3 months in early 2017.

The Theoretical Domains Framework (TDF) was used to guide the study, including data collection and analysis. The TDF was developed to research barriers to evidence implementation and is useful for providing context-based explanations as to why implementation of evidence-based practice is not being achieved. ${ }^{6}$ An interview guide was developed and pre-tested to ensure all domains of the TDF were addressed. Interviews lasted approximately one hour and were recorded then transcribed verbatim. Prior to data analysis all interview transcriptions were returned to the participants for review; no changes were requested.

Data analysis used deductive content analysis which is suitable for use with a structured matrix like the TDF. ${ }^{7}$ Following familiarisation with the data a coding manual was developed that mapped to the fourteen conceptual domains of the TDF. Text that did not fit with any domains was coded as 'other relevant'. The coded data was then refined, and themes identified from within the TDF or 'other relevant' category. This study was undertaken as part of a Masters degree and the first author is employed as a Quality and Effectiveness Lead with responsibility for improving adherence to the SSCB. Research and Development authorisation (reference number 2016CV07) and University Ethics approvals (UREC Application Number: 2016007_Lancaster) were obtained for this study. Informed consent was obtained prior to the interviews and all data was anonymised and stored securely.

Results

Coding identified 1596 statements relating to the implementation of the SSCB which were distributed across all fourteen TDF domains. Sixty three percent of these $(n=1005)$ were coded to six domains (Figure 1) which were prioritised for analysis.

[figure 1]

\section{Reinforcement}

Reinforcement related to anything that participants felt would or would not reinforce implementation of the SSCB and was the most frequently discussed domain (16\% of statements). There was overwhelming agreement that the evidence base around the individual parts of the SSCB and positive patient outcomes encouraged implementation.

"they are actually based on good clinical evidence [...], we know that these things actually make a difference to people." (Interviewee 8)

Another commonly held view was that establishing and reviewing the reasons for any patient not receiving all elements of the SSCB was worthwhile. Most participants suggested that one way to motivate staff to attain SSCB compliance would be to regularly display information on 'how we are doing'. Other individual areas were identified as positive reinforcers, for example the use of a 'nurse coordinator' which participants identified as increasing the likelihood of SSCB implementation.

Participants also identified negative reinforcers, the most prevalent of which was a belief that stroke care is not taken seriously enough at a macro level. The absence of noticeable political or organisational support was seen to contribute to the overall impression that stroke care is not considered to be an important field in modern healthcare and considered to be less important than other medical specialities. None of the staff 
interviewed felt that there was any real consequence for the organisation for either achieving or failing SSCB implementation.

"If we fail nothing really comes of it. We've had visits from the Scottish Government who told us to get a grip and what's come of that [...]" (Interviewee 5)

Environmental context and resources

Availability of resources along with the environment in which the SSCB is being implemented emerged as a key issue for this domain. Frequently discussed was lack of capacity across the system (NHS and social care perspectives) and the consequential pressures that this puts on the use of stroke beds.

Linked to this, participants frequently commented on staffing pressures both generally and in relation to availability of specialist stroke consultant staff. Some participants also discussed the increasing numbers of junior medical and nursing staff and reflected that their lack of experience and confidence made SSCB implementation challenging.

Two of the most striking barriers to SSCB implementation voiced across all eight interviews were environmental; firstly, an organisational culture that is not supportive of stroke care and secondly a lack of ownership of the SSCB. Relating to organisational culture, a commonly held view was that there was little managerial or organisational support for the efforts participants were making to improve the SSCB's implementation and stroke care in general.

"I think there needs to be more involvement from higher up, I don't know who higher up but people to help us meet the bundle" (Interviewee 3).

Linked to this, one participant suggested that a lack of political interest in stroke care directly influenced the organisation's interest.

"The support at political level for stroke has dropped [...] Fashions change and I think that the foot has been taken off the political gas [...] but that will change again because something will happen, [...] and the foot will go back on the gas because stroke is undoubtedly a major player in Scottish health [...]" (Interviewee 1)

A commonly held view of participants was that stroke care is not taken seriously because it is not seen as a medical emergency.

"Stroke is an emergency. Unless you have people who believe that, [...] until everybody treats stroke as a medical emergency [...] We're not able to do it." (Interviewee 4).

One participant suggested that the reason for this is that stroke care is not a medical speciality in its own right.

The lack of SSCB ownership came through in all interviews. Participants highlighted that the SSCB is composed of elements that happen at differing times during the hyperacute care phase, so different staff groups are responsible for their implementation. Consequently, this impacts on the way other areas perceive their role in implementation. Participants regularly discussed the challenges faced in engaging staff from the other areas and all agreed that without this engagement consistent implementation was extremely challenging.

"different people are responsible for different bits which is part of the problem. Part of the challenge. [...] so we are relying on other people. [...] it is a chain that involves so many different departments and so many individuals and you kind of need all bits of that chain to work for every patient." (Interviewee 2)

Participants identified a potential solution in this area: to implement direct admission to their stroke units. This would resolve inconsistencies with achieving the SSCB.

"Direct admissions would solve it, yes, it would solve a lot of these problems, I think it would solve everything." (Interviewee 3)

Knowledge 
Noticeably, none of the participants could tell the interviewer exactly what the SSCB consisted of, with participants frequently forgetting or incorrectly providing timescales and target percentages. Commonly during interviews participants had to be prompted to discuss the 'admission to a stroke unit' element. Initially it seemed that they did not think of this as a core part of the SSCB. However, as the interviews and analysis progressed it emerged that they understood this but felt powerless to influence this component of the SSCB.

There was also a lack of knowledge in relation to the evidence base around the SSCB with some participants stating they knew there was some (none had read it) and others stating they knew there was evidence for some of the components, but they were unaware of any specifically related to the SSCB. Regardless of this all participants stated they were aware of the benefits of the SSCB in terms of improved outcomes for patients.

"There is evidence that it has a better outcome, I can't remember all the figures but it does show that if it's implemented straight away and properly then there is a better chance of recovery than there is if it isn't" (Interviewee 7)

The importance of having the knowledge to correctly diagnose a stroke in the first place was repeatedly mentioned by all participants. This was seen to be essential to ensure appropriate SSCB application. Participants often reflected that general basic education about the SSCB was lacking and that if its importance was more widely understood and acknowledged then people would be more willing to ensure its implementation.

\section{Optimism}

Throughout the interviews the overwhelming feedback received was one of pessimism. Participants felt that consistent implementation of the SSCB for every person in the current climate was unachievable. Underpinning this was a lack of power to influence change.

"I think it is really challenging at times to feel that you have the power to make a difference to these things" (Interviewee 8) "that's part of the challenge there is not one single thing that I think we can do now that is going to fix it...." (Interviewee 2)

Participants were extremely negative about their ability to get all relevant patients into a stroke unit in a timeous manner

"there isn't enough beds and people don't move quickly enough so there is no capacity" (Interviewee 1)

The challenges linked to discharging patients meant that participants could see no way to get patients moved when their stroke units had reached capacity.

Participants also repeatedly discussed the lack of support from management and the organisation in general which again led them to believe that they were powerless to influence changes which they felt were necessary. There was also a generalised belief that staff from other areas would never see the SSCB as a priority, given all the other competing priorities that they faced. Two participants went further, questioning the use of a bundle with targets and whether targets are helpful. They felt that each of the SSCB components should be a routine part of good quality stroke care, suggesting that if the care provided was good then the SSCB would be achieved.

On a positive note, all participants were optimistic that given the right organisational support it would be possible in the future to implement the SSCB consistently. This support included stroke care being prioritised, progressing direct access to stroke units, working towards a $24 / 7$ stroke service and support with unblocking stroke units' exit strategy issues.

\section{Behavioural regulation}

Participants identified specific 'behavioural regulations' at a national, organisational or clinical team level. At a national level the SSCA was generally seen as something that had a positive effect on raising awareness of the SSCB and promoting its implementation. 
"because we know we are getting measured we do it and that probably is a big driver as much as the clinical care in terms of the fact you know you are going to get, [...] judged on whether you do it." (Interviewee 2)

However, there were mixed feelings about the Scottish Government's annual stroke visit, during which the SSCA results and stroke care standards are reviewed. Participants acknowledged that it raises awareness of stroke at an organisational level but felt there was no subsequent action, compounding feelings of powerlessness and reinforcing the feeling that stroke and the SSCB were not taken seriously at a macro level.

At an organisational level participants discussed factors that influenced their behaviour. Any form of organisational support or interest was welcomed but most participants perceived a lack of this. This is relevant to behavioural regulation because of the multiple areas and professionals who are involved with SSCB implementation. Participants believed that the lack of support promoted a system wide belief that the SSCB was not important and consequently that this affected behaviours.

At a clinical team level, participants agreed that feedback on the SSCB's implementation was important for practice. Those participants, in one hospital, who receive weekly feedback identified that this had a positive influence on SSCB implementation. Discussions revolved around exception reporting where any SSCB failures were investigated and followed up in an effort to drive improvement. Participants felt this could positively affect behaviour, but they recognised that grassroots staff do not routinely receive this feedback. When they do, behaviour change seems to be evident. All participants suggested that providing consistent feedback at a grassroots level, across the stroke pathway, could positively influence SSCB implementation.

One team was trying to identify a nurse to undertake a daily stroke coordination role, to promote compliance with the Scottish stroke care standards. This was found to be hugely influential in changing behaviour and increasing compliance. However, because of the current staffing complement the role was not consistently sustainable.

All participants agreed that direct admission to their stroke unit would ensure behaviour change because it would be specialist stroke staff driving SSCB implementation.

"I think we would need to admit straight to the stroke unit in an ideal world. I mean why are we putting patients through ED, AMU and here? Why are we doing that?" (Interviewee 4)

Noticeably, when discussing behavioural regulation participants started to suggest simple practical changes that could promote implementation. Given the opportunity to reflect on SSCB implementation prompted realisation that there were straightforward solutions, which might improve compliance.

\section{Beliefs about consequences}

When discussing their beliefs about the consequences of whether or not the SSCB was implemented the common theme was that stroke patients who did not receive the SSCB potentially suffered harm.

"I think not meeting these targets probably results in some harm. [...] I'm sure there are people who come to harm because of this." (Interviewee 5).

Three specific areas were highlighted: person-centred care; swallow screening and lack of stroke consultant cover. Given that the SSCB should be consistently applied to anyone suspected of suffering a stroke there were some concerns voiced that it reduced person-centred care i.e. on occasion people could be scanned unnecessarily or have an extra ward move in their patient journey. All who mentioned this nevertheless felt that the benefits of receiving the SSCB outweighed the risks posed. Of greater concern was that staff in both hospitals' emergency departments refused to undertake swallow screening. This was raised as potentially harmful and was mentioned frequently by all participants.

"They may be [...] kept nil by mouth when they could be allowed to eat and drink [...], or they could be given water to drink when their swallow is unsafe, and they could be silently aspirating [...] for years we have been trying to get them (ED) to do it (Swallow screening) and they won't." (Interviewee 5) 
Finally, in one of the hospitals the lack of specialist stroke consultant cover was felt by the participants from that hospital as having negative consequences.

"I mean just now we don't even have a consultant ward round every day [...] I think just now I feel that patients are not getting the right interventions that they need at the right time" (Interviewee 4).

In relation to this domain personal consequences also emerged in the interviews. The overwhelming personal consequence for participants was emotional; they felt 'bad' and 'guilty'. This related to times when the SSCB was not implemented, even though they were aware of the evidence based for its implementation and the potential harmful consequences to the patient. They also felt 'frustrated' because of feelings of powerlessness and their inability to make the changes they felt were needed to ensure consistent SSCB implementation. All participants suggested that there were no other personal consequences apart from perhaps occasionally being asked why a patient had not received the SSCB. Most participants also discussed feelings of satisfaction when their patients did receive it.

\section{Discussion}

The main purpose of this study was to explore the enablers and barriers to the implementation of the SSCB in one Scottish Health Board. This is the first study to specifically consider staffs' perceptions and experiences of SSCB implementation. The key areas that emerged during the analysis as increasing the likelihood of patients receiving the SSCB in this Health Board relate to knowledge, resources and organisational culture.

\section{The significance of knowledge}

Knowledge was a key theme throughout all interviews and was discussed by participants across several domains. The most surprising finding was the lack of knowledge amongst participants of precisely what the SSCB consisted of. Although all participants knew the four component areas none described all the targets and timescales accurately. Van Achterberg et al suggest that there is frequently a gap between knowledge and practice. ${ }^{8}$ An important example of this was that during the analysis it was clear that participants were not, unless prompted, discussing 'getting patients to the stroke unit'. It seemed that they lacked knowledge about this component of the SSCB. However, initially they had all discussed admission to a stroke unit as being part of the SSCB and most had also highlighted its importance. This gap between knowledge and practice appears to be due to the lack of control participants had over 'entry to stroke units'; they simply forgot about this being a key component of SSCB implementation.

When looking for evidence to support this everything appeared to link forgetfulness to stress though there was no evidence noted during the interviews or analysis to support this theory. This could potentially be an interesting area for further research.

An enabler often suggested by participants was the development of regular short training sessions to raise awareness of the importance of the SSCB. The importance of training in stroke care is nationally recognised in Scotland and Priority 4 of the 'Stroke Improvement Plan' states that staff should be trained to an appropriate level. ${ }^{9}$ The e-learning Stroke Training and Awareness Resources (STARS) 'Core Competencies' are considered to be the minimum nationally approved courses ${ }^{4}$ however, the SSCB is only mentioned very briefly in STARS. The lack of recognition of the SSCB in the national training database may in itself be compounding the general lack of knowledge and priority placed on the SSCB. This would be a relatively easy area to address with the development of a core competency around the SSCB and the current research base for stroke care.

Knowledge was also sought by participants in terms of their eagerness to receive consistent feedback at a ward level on how they were doing with implementing the SSCB. As most participants felt that grassroots feedback, would enhance SSCB implementation. The necessity of knowledge is also recognised in other areas e.g. The Report of the Mid Staffordshire NHS Foundation Trust which highlighted the importance of care being provided by those with relevant knowledge. ${ }^{10}$

The influence of resources 
Resources were a recurring theme throughout all interviews and across all 14 domains. Discussions focussed on three main areas: staffing, the current stroke pathway and general system capacity. In terms of staffing the commonly discussed barriers related to a general lack of availability of specialised stroke care staff and particularly medical stroke specialists. Lack of appropriate staffing is a commonly discussed key barrier in much of the stroke and evidence-based practice literature (EBP). ${ }^{11},{ }^{12},{ }^{13}$ Furthermore the Vale of Leven ${ }^{14}$ and Mid Staffordshire ${ }^{11}$ enquiries emphasise the unacceptability of the lack of appropriate staffing, in relation to quality of care and patient safety.

Participants suggested that a nurse coordinator who would identify stroke patients on admission to hospital would ensure that they received the SSCB. Drury et $\mathrm{al}^{15}$ found that three quarters of the stroke units they studied had a member of staff whose role was the dissemination and implementation of stroke guidelines, highlighting that:

- the maintenance of the role was compromised due to a lack of staffing resource

- when these roles were adequately staffed then guidelines were successfully implemented and sustained

The findings from Dale's ${ }^{11}$ study mirror the findings of this study and consequently a key improvement area should be consideration of how to develop this role in a sustainable way.

The current stroke pathway was highlighted repeatedly as a key barrier to SSCB implementation because of the number of services and staff involved. Participants suggested that the current pathway encouraged a lack of ownership of the SSCB, contributing to a lack of collaboration and encouraging an organisational culture that did not prioritise its importance. All participants considered direct admission to their stroke units as the obvious solution to this and moreover direct admission was the most discussed enabler to the implementation of the SSCB. Improving the pathway to allow direct admission to the stroke units would require a substantial amount of organisational change which may or may not be financially viable. Donnellan et al found that finance for the development and maintenance of stroke services was a barrier in their healthcare setting. ${ }^{16}$

Locally stroke patients admitted via AMU are the same patients who would be directly admitted to the stroke units. If they then consistently received all the elements of the SSCB Turner et al's evidence suggests that their outcomes would be improved thus potentially offering a cost saving over the longer term. ${ }^{17}$ This is particularly relevant as this evidence of improved outcomes is the only evidence to date on the SSCB.

The third area most discussed in resources related to capacity and flow in the stroke units with lack of beds and exit strategies regularly cited as barriers. The Scottish Government has signed up to a 'Whole Systems Patient Flow Improvement Programme' aimed at adopting an approach to patient flow which will ensure patients receive 'the right care at the right time in the right place by the right team every time.' Locally, in this Health Board area, one of the key transformation programmes is Capacity and Flow however stroke was not part of this Programme. Purvis et al cite limited stroke unit beds as a key barrier to getting patients into their stroke unit. ${ }^{18}$ This was often also highlighted in this study in relation to the use of stroke unit beds for boarders and delayed discharges

The influence of organisational culture

Organisational culture was the area that presented itself most strongly as a barrier to the implementation of the SSCB. Participants consistently discussed the lack of support they experienced as leading to feelings of helplessness as to how things could be improved. This lack of support manifested itself at both a national and local level. Medical staff wondered whether the concentration on an acute care bundle was diluting the importance of stroke care in general and none of the staff had experienced any genuine form of recognition whether or not the SSCB was achieved. This links to a key finding in both the Vale of Leven and Mid Staffordshire enquiries. They found organisational cultures which accepted poor standards of care and tolerated the risk this posed to patients. ${ }^{10,14} \mathrm{~T}$ he Vale of Leven enquiry found a "mismatch between expectation and implementation" and highlighted a management approach that demonstrated an organisational culture that viewed infection prevention and control as being of low priority. In this study participants frequently verbalised their frustration that stroke care and the SSCB were seen as low priority. Other studies into 
implementation of stroke standards and EBP also found lack of support to be a major barrier. ${ }^{15,19}$ Hardy et al found that when leaders prioritised EBP by promoting organisational culture that EBP improved..$^{20}$

This lack of support led to feelings of helplessness, powerlessness and frustration alongside pessimism about their ability to influence the changes required to ensure consistent SSCB implementation. Kanter suggests that powerlessness is caused by an organisations inability to provide three key components of power, namely: information, resources and support ${ }^{21}$ and Gary believes that disempowerment follows when any of these three components is missing as this leads to an inability to provide adequate patient care. ${ }^{22}$ Interestingly these three components are the three key themes which emerged as the areas which appear to have considerably influenced the effectiveness of the implementation of the SSCB in this Health Board area.

\section{Limitations}

Study limitations have been recognised. The data collection and analysis were carried out by a single investigator who is involved with stroke improvement work therefore increasing the potential for subjectivity in the analysis and discussion of findings. To mitigate this the study was supervised by two experienced researchers and the findings were sent to participants for feedback to reduce this potential.

This was a small study with only eight participants from one Health Board. Its size limits transferability as it focuses on the experiences of this limited group of participants. Service users' perspectives of SSCB implementation were not sought. Those interviewed suggested that most patients have no awareness of the existence of the SSCB. Ideally service users' experience should be included in any future research in this area.

\section{Conclusion}

This study, the first of its kind, aimed to explore the views of nursing and medical staff in relation to the enablers and barriers to implementation of the SSCB. The findings potentially offer a focus for the optimisation of not only the implementation of the SSCB but also other national improvement projects in the future. The findings are also potentially relevant to those with an improvement role in Scottish hospitals looking to increase local compliance with the SSCB and should be carefully considered for contextual relevance within their own area. As this is the first comprehensive qualitative assessment of the enablers and barriers to the implementation of the SSCB further research will be required, potentially at a national scale, to confirm or challenge this study's findings and promote further understanding. Further evidence could ensure a more consistent application of the SSCB. Consideration should be given to the development of a simple education programme about the SSCB and the evidence base which supports it. The development of permanent stroke nurse coordinator roles or alternatively direct admission to stroke units should also be considered. Stroke care should be prioritised both locally and nationally and this should be evidenced through stroke having a significant presence in local and national governance agendas.

\section{References}

1. Cane J, O'Connor D, Michie S. Validation of the theoretical domains framework for use in behaviour change and implementation research. Implementation Science . 2012;7(1):7-37. doi:10.1186/1748-5908-7-37

2. State of the Nation: stroke statistics. Stroke Association. https://www.stroke.org.uk/resources/statenation-stroke-statistics. Published 2017. Accessed October 12, 2016.

3. Scottish Stroke Care Audit Team. (2015). Scottish Stroke Care Audit, 2015 National Report, Stroke Services In Scottish Hospitals . Edinburgh: ISD Scotland Publications; 2015. http://www.strokeaudit.scot.nhs.uk/Downloads/2015_report/SSCA-report-2015-web.pdf. Accessed May 10, 2016.

4. Scottish Stroke Care Audit Team. Scottish Stroke Improvement Programme, 2017 Report . Edinburgh: ISD Scotland Publications; 2017. http://www.strokeaudit.scot.nhs.uk/Publications/docs/2017-0711-SCCA-Report.pdf. Accessed October 12, 2017. 
5. Scottish Stroke Care Audit Team. Scottish Stroke Improvement Programme, 2018 Report . Edinburgh: ISD Scotland Publications; 2018. http://www.strokeaudit.scot.nhs.uk/Publications/docs/2018-07-10-SSCAReport.pdf. Accessed October 12, 2018.

6. Michie S. Making psychological theory useful for implementing evidence based practice: a consensus approach. Quality and Safety in Health Care . 2005;14(1):26-33. doi:10.1136/qshc.2004.011155

7. Elo S, Kyngas H. The qualitative content analysis process. J Adv Nurs . 2008;62(1):107.

8. Van Achterberg T, Schoonhoven L, Grol R. Nursing Implementation Science: How Evidence-Based Nursing Requires Evidence-Based Implementation. Journal of Nursing Scholarship . 2008;40(4):302-310. doi:10.1111/j.1547-5069.2008.00243.x

9. Scottish Government. Stroke Improvement Plan . Edinburgh: Scottish Government; 2014.

10. Department of Health Report Of The Mid Staffordshire NHS Foundation Trust Public Inquiry.pg 76. London: The Stationary Office Limited; 2013.

11. Dale S, Levi C, Ward J et al. Barriers and Enablers to Implementing Clinical Treatment Protocols for Fever, Hyperglycaemia, and Swallowing Dysfunction in the Quality in Acute Stroke Care (QASC) Project-A Mixed Methods Study. Worldviews Evid Based Nurs . 2015;12(1):41-50. doi:10.1111/wvn.12078

12. Donnellan C, Sweetman S, Shelley E. Health professionals' adherence to stroke clinical guidelines: A review of the literature. Health Policy . 2013;111(3):245-263. doi:10.1016/j.healthpol.2013.05.002

13. Schwamm L, Fonarow G, Reeves M et al. Get With the Guidelines-Stroke Is Associated With Sustained Improvement in Care for Patients Hospitalized With Acute Stroke or Transient Ischemic Attack. Circulation . 2009;119(1):107-115. doi:10.1161/circulationaha.108.783688

14. MacLean T. Final Report. Vale Of Leven Hospital Inquiry . APS Group; 2014. http://www.valeoflevenhospitalinquiry.org/report.aspx. Accessed September 20, 2016.

15. Drury P, McInnes E, Hardy J, Dale S, Middleton S. Stroke unit Nurse Managers' views of individual and organizational factors liable to influence evidence-based practice: A survey. Int $J$ Nurs Pract . 2015;22(2):169-178. doi:10.1111/ijn.12396

16. Donnellan C, Sweetman S, Shelley E. Implementing clinical guidelines in stroke: A qualitative study of perceived facilitators and barriers. Health Policy . 2013;111(3):234-244. doi:10.1016/j.healthpol.2013.04.002

17. Turner M, Barber M, Dodds $\mathrm{H}$ et al. Implementing a Simple Care Bundle Is Associated With Improved Outcomes in a National Cohort of Patients With Ischemic Stroke. Stroke . 2015;46(4):1065-1070. doi:10.1161/strokeaha.114.007608

18. Purvis T. Implementation of evidence- based stroke care: enablers, barriers, and the role of facilitators. $J$ Multidiscip Healthc . 2014;7:389

19. Prior M, Guerin M, Grimmer-Somers K. The effectiveness of clinical guideline implementation strategies - a synthesis of systematic review findings. J Eval Clin Pract . 2008;14(5):888-897. doi:10.1111/j.13652753.2008.01014.x

20. Hardy A, Nevin-Woods C, Proud S, Brownson R. Promoting Evidence-Based Decision Making in a Local Health Department, Pueblo City-County, Colorado. Prev Chronic Dis . 2015;12. doi:10.5888/pcd12.140507

21. Kanter R. The enduring skills of change leaders. Leader to Leader . 1999;1999(13):15-22. doi:10.1002/ltl.40619991305

22. Gary D. The Why and Wherefore of Empowerment: The Key to Job Satisfaction and Professional Advancement. Nurs Forum . 2002;37(3):33-36. doi:10.1111/j.1744-6198.2002.tb01008.x 
Figure 1: Statements coded to TDF domains for all interviews (number per domain and cumulative percentage)
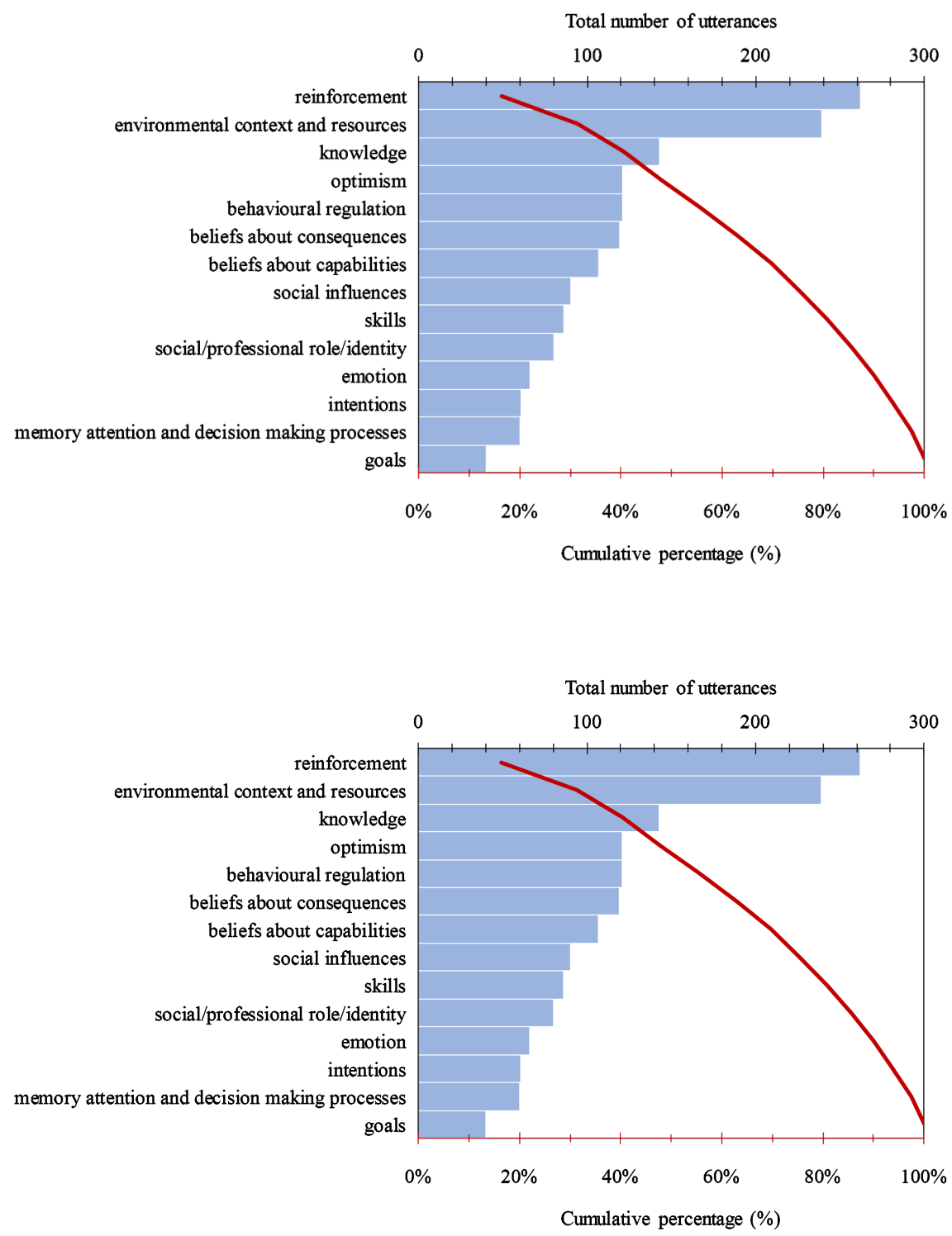